\title{
Tailoring magnetic behavior in the Tb-Au-Si quasicrystal approximant system
}

Girma H. Gebresenbut, ${ }^{\dagger,}{ }^{*}$ Mikael S. Andersson, ${ }^{\ddagger}$ Per Nordblad, ${ }^{\ddagger}$ Martin Sahberg, ${ }^{\dagger}$ and Cesar Pay Gómez $^{\dagger}$

${ }^{\dagger}$ Department of chemistry-Ångström laboratory, Uppsala University, 75121 Uppsala, Sweden

${ }^{\ddagger}$ Department of Engineering Sciences-Ångström laboratory, Uppsala university, 75121 Uppsala, Sweden

KEYWORDS: Approximants of quasicrystals, high temperature synthesis, magnetic properties. 


\section{The 'arc-melting-self-flux' synthesis method}

Standard arc-melting and self-flux synthesis techniques were attempted to synthesize the ACs in the Tb-Au-Si system but they were not satisfactory. The arc-melting synthesis method can cast alloys in few seconds, removes oxidized surfaces from melted ingots and provide homogenous and shiny ingots. However, it is not the preferred method to grow large single crystals due to its fast cooling rate from the melt. Arc-melted and annealed $\mathrm{Tb}-\mathrm{Au}-\mathrm{Si} \mathrm{ACs}$ resulted in shiny and homogenous samples, but obtaining single crystals of size $\approx 10^{-3}$ $\mathrm{mm}^{3}$ appropriate for SCXRD measurements was almost impossible, even after 30 days of annealing at $750^{\circ} \mathrm{C}$. The self-flux synthesis method requires melting at least most of the reacting component and uses the melt as a flux to dissolve the high-melting part of the sample. When the temperature is slowly lowered near the nucleation point of a phase, crystals start to nucleate and grow in size. It is generally recommended to lower the cooling rate for better crystal growth. During the synthesis of Tb-Au-Si ACs using the self-flux technique; $\mathrm{Au}$ was the component with the lowest melting point (M.P. $\approx 1064{ }^{\circ} \mathrm{C}$ ). The molten Au was used as a flux to dissolve granules of $\mathrm{Tb}\left(\mathrm{M} . \mathrm{P} . \approx 1360{ }^{\circ} \mathrm{C}\right)$ and $\mathrm{Si}\left(\mathrm{M} . \mathrm{P} . \approx 1410{ }^{\circ} \mathrm{C}\right)$. The samples were heated to $1100{ }^{\circ} \mathrm{C}$ and slowly cooled below the melting points of the approximants and annealed at $750{ }^{\circ} \mathrm{C}$ for 30 days. It was possible to get single crystals appropriate for SCXRD measurements but highly demanding. The self-flux method will not be appropriate when the amount of flux is insufficient to ensure the complete dissolution of the high-melting components, especially in cases when the low melting component is not the majority constituent. Important technical details such as the thermal stability of the muffle furnaces and reaction vessels at very high temperatures should be considered during this kind of synthesis. For example, the stainless steel tubes used in these syntheses could hardly stays more than five hours at $1100{ }^{\circ} \mathrm{C}$ without being fractured.

The present synthesis technique, 'arc-melting-self-flux', stands to benefit from both arc-melting and self-flux methods. Homogenous, un-oxidized and phase pure samples that contain single crystals more than appropriate for SCXRD measurements were readily obtained. In this synthesis method the components are first arc-melted with the same process as the standard method. The arc-melted ingot undergoes a synthesis procedure similar to the self-flux technique. Unlike the standard self-flux technique however, the whole sample could melt completely at once if the ingot has a unique melting point, for example when we have a single phase ingot. Hence, it is necessary to modify the conventional temperature program used for self-flux method. First the sample is heated above the melting point of the majority component of the ingot; it is then slowly cooled below the nucleation point of the phase in focus and is annealed for a few hours. At this state, from a practical point of view, the sample could contain single crystals of different sizes which will melt at slightly different rates if they are heated slightly above their melting points. The sample will not be cooled down to room temperature as is the usual case in standard methods; it instead undergoes an original heat treatment procedure which we call 'sacrificial-crystal-growth'. The sample is re-heated slightly above or around its melting point in order to melt (sacrifice) some of the smaller crystallites. At this stage, the sample is comprised of larger solid crystals (seeds) and liquid melt, with similar chemical compositions. When the sample temperature slowly lowers across the nucleation point, the seeds are used as preferred crystal growth sites for the melt resulting in larger single crystals. The 'sacrificial-crystal-growth' procedure can be implemented several times to assure further crystal growth.

\section{Temperature program used for 'Arc-melting-self-flux' synthesis}

The samples were first heated to $950^{\circ} \mathrm{C}$ to assure re-melting of the ingots, cooled to $850^{\circ} \mathrm{C}$ during 1 hour and left at $850^{\circ} \mathrm{C}$ for 5 hours. After that, the samples were slowly cooled to $750^{\circ} \mathrm{C}$ during 17 hours to allow the nucleation and growth of crystals. At this stage what we call the 'sacrificial-crystal-growth' step was implemented: the samples were quickly heated to $850{ }^{\circ} \mathrm{C}$ during 2 hours, left there for 5 hours and slowly cooled to $750{ }^{\circ} \mathrm{C}$ during 17 hours. The purpose of the 'sacrificial-crystal-growth' is to re-melt some of the smaller crystals so that the bigger ones can be used as seeds for the consequent crystal growth. Therefore, oscillating the temperature slightly above and below the melting points of the ingots allows for a better 
crystal growth. The 'sacrificial-crystal-growth' was implemented three times. The ingots were annealed at $750^{\circ} \mathrm{C}$ for seven days to assure homogeneity of the samples. They were then cooled to $500^{\circ} \mathrm{C}$ during ten hours, left at $500^{\circ} \mathrm{C}$ for two days and cooled to room temperature during five hours.

Figure S1. Plots of a temperature program used for 'arc-melting-self-flux' synthesis technique, in order to prepare approximants in the Tb-Au-Si system.

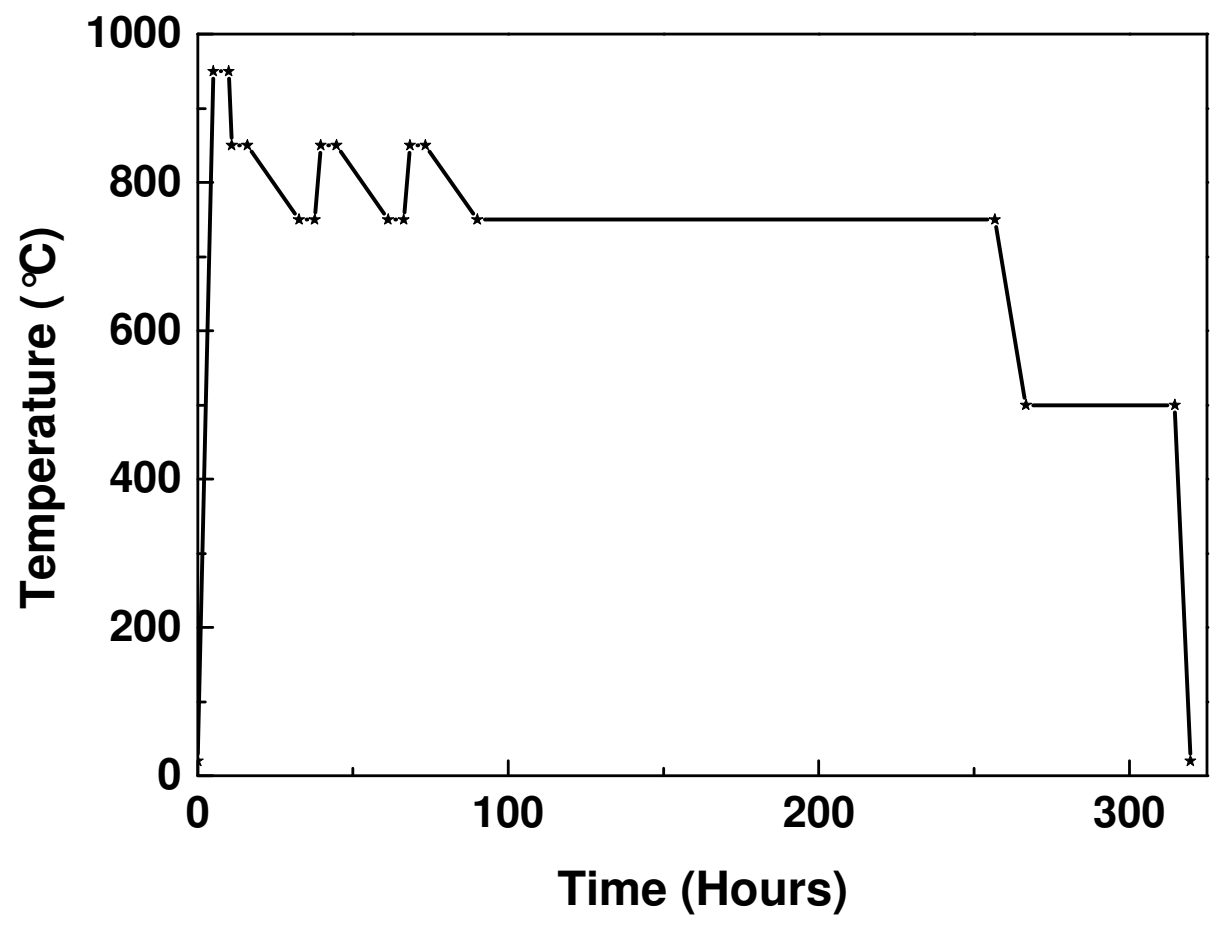


Table S1. Atomic parameters obtained from SCXRD refinement. Site occupancy factor (S.O.F.) and Wyckoff positions (Wyck.) are indicated. All atomic positions are standardized.

\begin{tabular}{|c|c|c|c|c|c|c|c|}
\hline Atom & Wyck. & Compound & S.O.F. & $\mathbf{x} / \mathbf{a}$ & $\mathrm{y} / \mathbf{b}$ & $\mathrm{z} / \mathbf{c}$ & $\mathrm{U}_{\text {eq. }}\left[\AA^{2}\right]^{\dagger}$ \\
\hline \multirow{5}{*}{ Aur } & \multirow{5}{*}{$48 \mathrm{~h}$} & TAS(o) & 1 & $0.10683(3)$ & $0.33874(3)$ & $0.20112(3)$ & 0.01523 \\
\hline & & $\operatorname{TAS}(40)$ & 1 & $0.10696(12)$ & $0.33852(12)$ & $0.2005(1)$ & 0.0317 \\
\hline & & $\operatorname{TAS}(52)$ & 1 & $0.10754(15)$ & $0.33795(16)$ & $0.20036(16)$ & 0.0469 \\
\hline & & $\operatorname{TAS}(70)$ & 1 & $0.10773(15)$ & $0.33704(14)$ & $0.19982(15)$ & 0.04617 \\
\hline & & TAS(100) & 1 & $0.1072(2)$ & $0.3364(2)$ & $0.1990(2)$ & 0.051 \\
\hline \multirow{5}{*}{ Au2 } & \multirow{5}{*}{$24 \mathrm{~g}$} & TAS(o) & 1 & o & $0.4022(9)$ & $0.35592(11)$ & 0.0244 \\
\hline & & $\operatorname{TAS}(40)$ & 1 & o & $0.40289(18)$ & 0.35527 & 0.0393 \\
\hline & & $\operatorname{TAS}\left(5^{2}\right)$ & 1 & o & $0.4029(2)$ & 0.35510 & 0.04713 \\
\hline & & TAS(7o) & 1 & o & $0.40259(17)$ & $0.3545^{\circ}$ & 0.04553 \\
\hline & & TAS(100) & 1 & $\mathrm{o}$ & $0.4030(3)$ & 0.35410 & 0.05233 \\
\hline \multirow{5}{*}{$\mathrm{Au}_{3}$} & \multirow{5}{*}{$12 \mathrm{~d}$} & TAS(o) & 1 & $0.40425(14)$ & o & $\mathrm{O}$ & 0.04457 \\
\hline & & $\operatorname{TAS}(40)$ & 1 & 0.40530 & o & o & 0.0545 \\
\hline & & $\operatorname{TAS}\left(5^{2}\right)$ & 1 & 0.40350 & o & o & 0.0601 \\
\hline & & $\operatorname{TAS}(70)$ & 1 & 0.40270 & o & o & 0.05803 \\
\hline & & TAS(100) & 1 & 0.40190 & o & o & 0.0572 \\
\hline \multirow{5}{*}{$\mathrm{Au}_{4} / \mathrm{Au}_{5}$} & \multirow{5}{*}{$16 f$} & TAS(o) & $1 / 0$ & $0.15018(8)$ & $0.15018(8)$ & $0.15018(8)$ & 0.0254 \\
\hline & & $\operatorname{TAS}(40)$ & $0.933 / 0.067$ & $0.1466(2) / 0.1799(14)$ & $0.1466(2) / 0.1799(14)$ & $0.1466(2) / 0.1799(14)$ & 0.0509 \\
\hline & & $\operatorname{TAS}\left(5^{2}\right)$ & $0.918 / 0.082$ & $0.1454(2) / 0.1801(13)$ & $0.1454(2) / 0.1801(13)$ & $0.1454(2) / 0.1801(13)$ & 0.0613 \\
\hline & & $\mathrm{TAS}(70)$ & $0.894 / 0.106$ & $0.1431(2) / 0.1801(9)$ & $0.1431(2) / 0.1801(9)$ & $0.1431(2) / 0.1801(9)$ & 0.062 \\
\hline & & TAS(100) & $0.887 / 0.112$ & $0.1409(3) / 0.1793(11)$ & $0.1409(3) / 0.1793(11)$ & $0.1409(3) / 0.1793(11)$ & 0.0624 \\
\hline \multirow{5}{*}{ Au6/Si6 } & \multirow{5}{*}{$24 \mathrm{~g}$} & TAS(o) & $0.547 / 0.453$ & $\mathrm{O}$ & $0.23589(17)$ & $0.07924(17)$ & 0.0233 \\
\hline & & $\operatorname{TAS}(40)$ & $0.576 / 0.424$ & o & $0.23377(16)$ & 0.08073 & 0.04587 \\
\hline & & $\operatorname{TAS}\left(5^{2}\right)$ & $0.55 / 0.45$ & o & $0.2312(4)$ & 0.07930 & 0.0544 \\
\hline & & $\operatorname{TAS}(70)$ & $0.542 / 0.45^{8}$ & o & $0.2273(4)$ & 0.07790 & 0.05877 \\
\hline & & TAS(100) & $0.413 / 0.587$ & o & $0.2241(6)$ & 0.07350 & 0.05183 \\
\hline \multirow{5}{*}{$\mathrm{Au} 7 / \mathrm{Si} 7$} & \multirow{5}{*}{$12 \mathrm{e}$} & TAS(o) & $0.238 / 0.762$ & $0.2030(4)$ & $\mathrm{O}$ & 0.5 & 0.02117 \\
\hline & & $\operatorname{TAS}(40)$ & $0.345 / 0.655$ & $0.1985(5)$ & o & 0.5 & 0.03453 \\
\hline & & $\operatorname{TAS}\left(5^{2}\right)$ & $0.334 / 0.666$ & $0.1995(3)$ & o & 0.5 & 0.041 \\
\hline & & $\operatorname{TAS}(70)$ & $0.356 / 0.644$ & 0.19930 & o & 0.5 & 0.04313 \\
\hline & & TAS(100) & $0.265 / 0.735$ & 0.19630 & o & 0.5 & 0.03967 \\
\hline \multirow{5}{*}{ Au8/Si8 } & \multirow{5}{*}{$24 \mathrm{~g}$} & TAS(o) & $0.216 / 0.117$ & o & $0.0853(5)$ & $0.0605(6)$ & 0.0484 \\
\hline & & $\operatorname{TAS}(40)$ & $0.118 / 0.082$ & $\mathrm{o}$ & 0.08400 & $0.0535(8)$ & 0.069 \\
\hline & & $\operatorname{TAS}(52)$ & $0.106 / 0.055$ & o & 0.08380 & $0.054(1)$ & 0.079 \\
\hline & & $\operatorname{TAS}(70)$ & $0.065 / 0.035$ & o & $0.0841(19)$ & $0.0541(14)$ & 0.085 \\
\hline & & TAS(100) & - & - & - & - & - \\
\hline \multirow{5}{*}{ Si9 } & \multirow{5}{*}{$8 c$} & TAS(o) & 1 & 0.25 & 0.25 & 0.25 & 0.0237 \\
\hline & & $\operatorname{TAS}(40)$ & 0.933 & 0.25 & 0.25 & 0.25 & 0.037 \\
\hline & & $\operatorname{TAS}\left(5^{2}\right)$ & 0.918 & 0.25 & 0.25 & 0.25 & 0.049 \\
\hline & & TAS(7o) & 0.894 & 0.25 & 0.25 & 0.25 & 0.049 \\
\hline & & TAS(100) & 0.887 & 0.25 & 0.25 & 0.25 & 0.048 \\
\hline \multirow{5}{*}{ Tb1 } & \multirow{5}{*}{$24 g$} & TAS(o) & 1 & o & $0.18650(11)$ & $0.30533(11)$ & 0.01087 \\
\hline & & $\operatorname{TAS}(40)$ & 1 & o & $0.1857(2)$ & 0.30530 & 0.02453 \\
\hline & & $\operatorname{TAS}(52)$ & 1 & o & $0.1863(1)$ & 0.30535 & 0.03113 \\
\hline & & $\operatorname{TAS}(70)$ & 1 & o & $0.18654(8)$ & 0.30502 & 0.02943 \\
\hline & & TAS(100) & 1 & o & $0.1868(1)$ & 0.30459 & 0.03107 \\
\hline \multirow{5}{*}{ Tb2 } & \multirow{5}{*}{$2 \mathrm{a}$} & TAS(o) & - & - & - & - & - \\
\hline & & $\operatorname{TAS}(40)$ & 0.401 & o & o & o & 0.102 \\
\hline & & $\operatorname{TAS}\left(5^{2}\right)$ & 0.516 & o & o & o & 0.119 \\
\hline & & TAS(70) & 0.699 & o & o & o & 0.123 \\
\hline & & TAS(100) & 1 & o & o & o & 0.12 \\
\hline
\end{tabular}

†Ueq. $=\left(\mathrm{U}_{11}+\mathrm{U}_{22}+\mathrm{U}_{33}\right) / 3$. 
Figure S2. Concentric polyhedral cluster units in the cubic unit cell of (a) TAS(o), (b) TAS(40), (c) TAS(70) and (d) TAS(10o) ACs. Arranged from left to right are a disordered tetrahedron (c-Tb site), pentagonal dodecahedron, icosahedron, icosi-dodecahedron and (defect) rhombic triacontahedron. All atomic sites are represented with thermal ellipsoids.

(a)

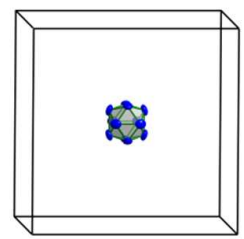

(b)

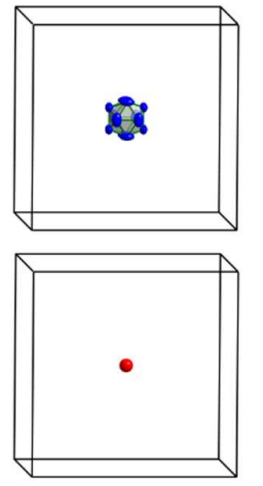

(c)
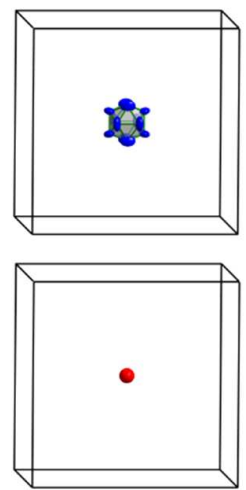

(d)
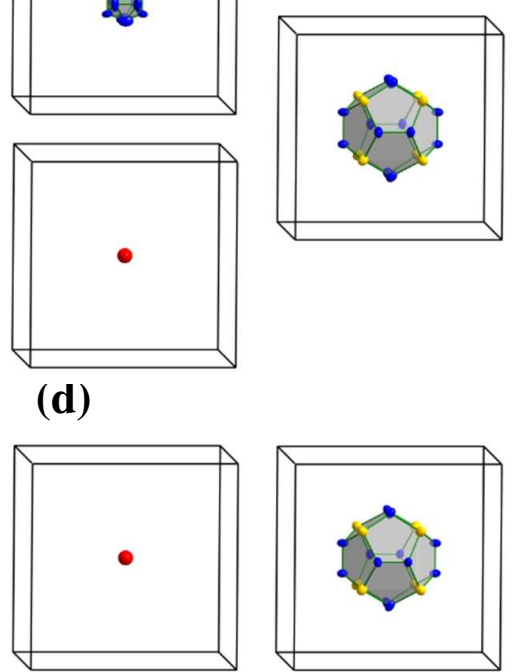
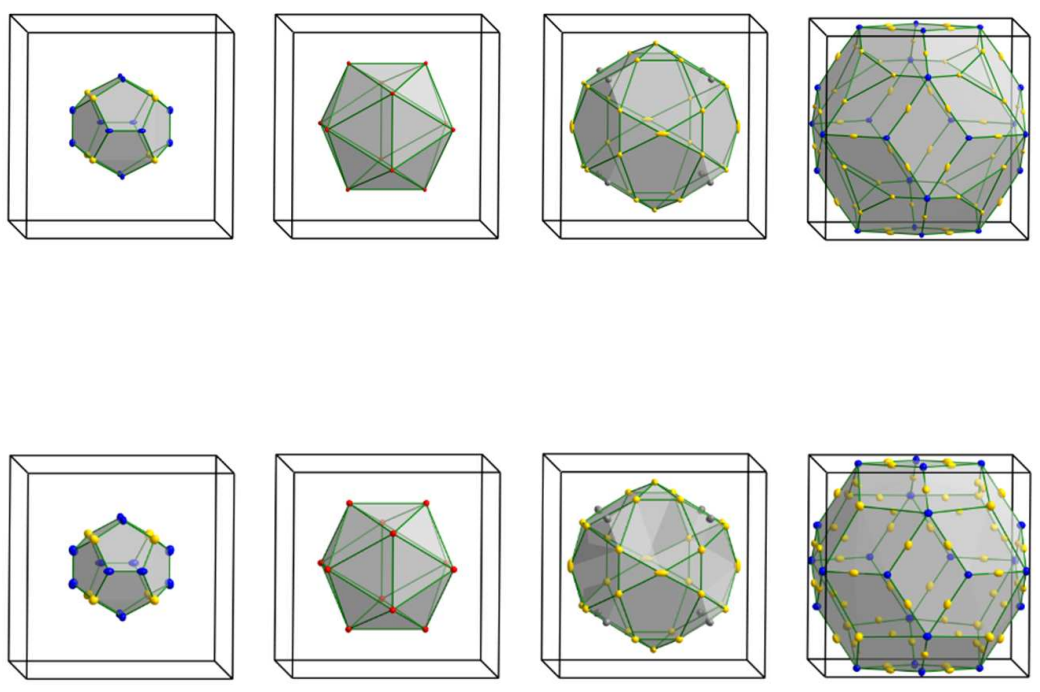
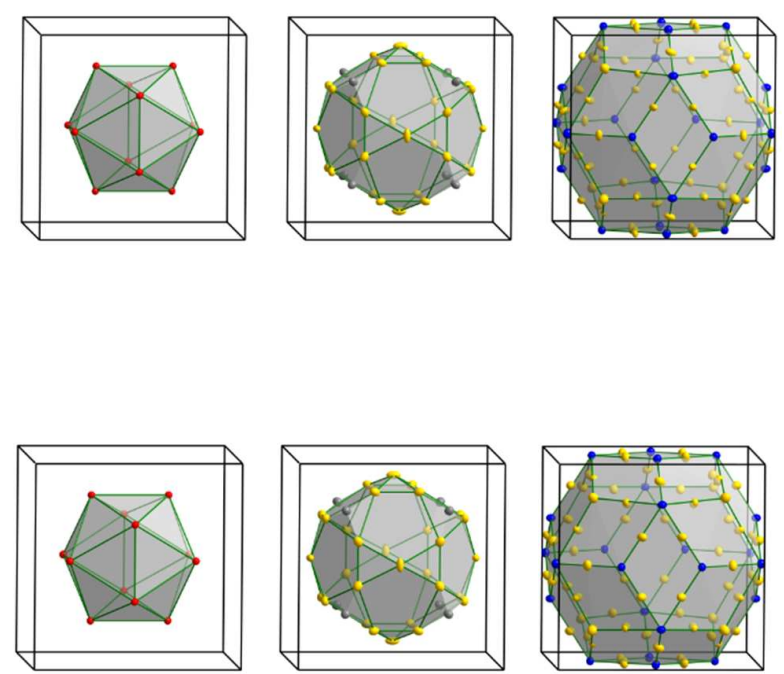
Figure S3: Experimental results obtained from as-casted Tb-Au-Si alloy with an intermediate chemical composition between TAS(o) and TAS(10o) compounds. (a) Observed PXRD intensities showing clear peak separations. The inset is a zoom in section highlighted with the red rectangle. (b) A backscattered SEM image showing a lamella-type microstructure.

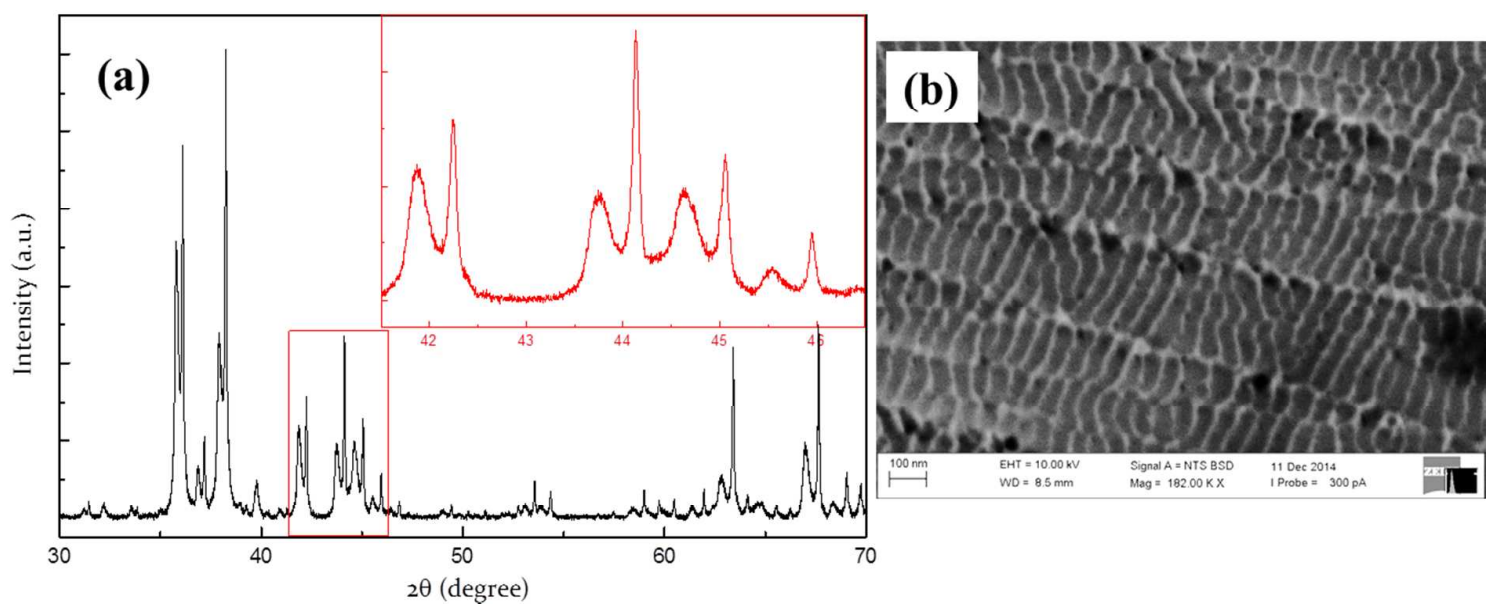


Figure S4. Magnetization vs temperature graph for samples (a) TAS(40), TAS(52) and (c) TAS(70). The insets in each plot are derivatives of FC curves vs temperature.

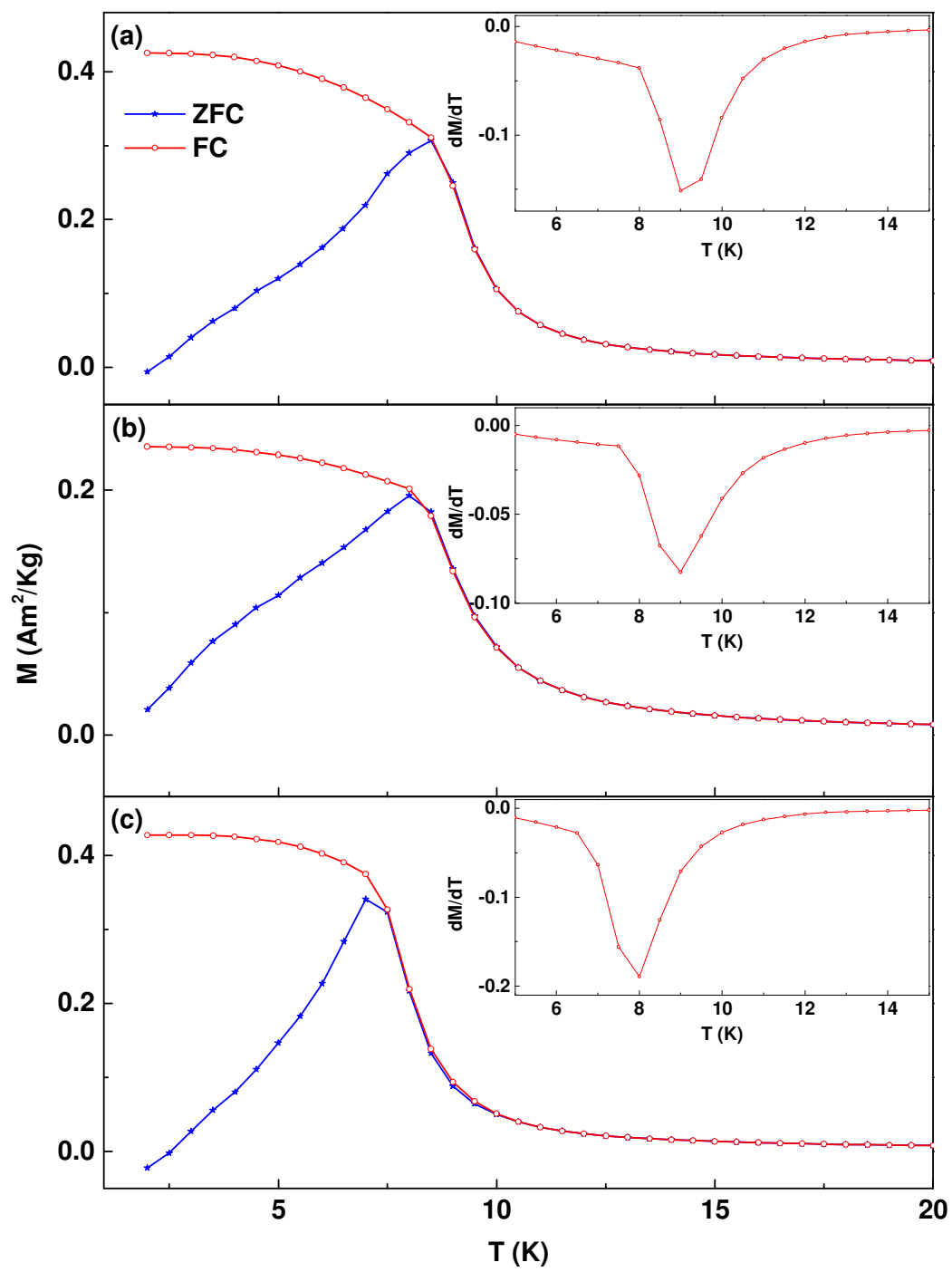

